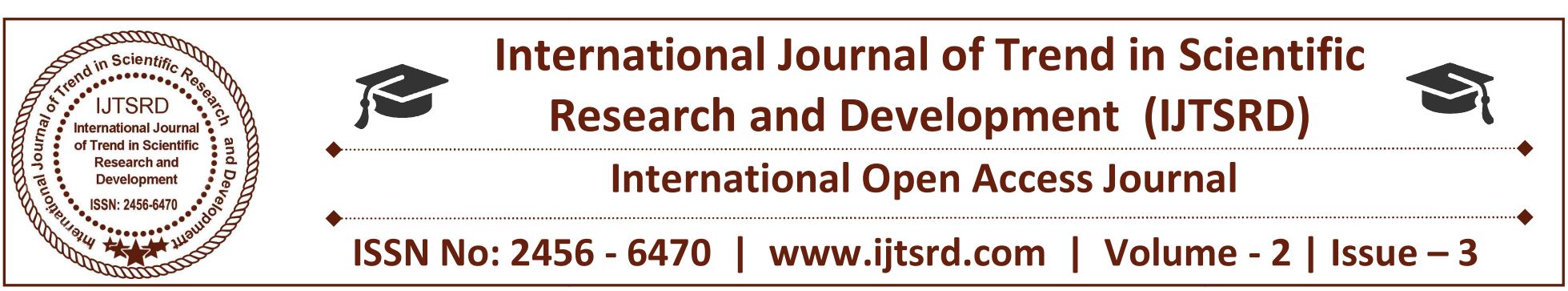

Popular Article

\title{
Phosphorus Deficiency Phosphorous Deficiency in Animals
}

\author{
${ }^{1}$ Abhivyakti Pathak, ${ }^{2 *}$ Manish Kumar Verma, ${ }^{3}$ Rajat Verma, ${ }^{4}$ Shishu Pal Singh \\ ${ }^{1-3}$ Research Scholar, G. B. Pant University of Agriculture and Technology, Pantnagar \\ ${ }^{4}$ Department of Agriculture, Krishi Bhavan, Varanasi
}

Phosphorus deficiency is a condition, in which Phosphorus is insufficient or is not utilized properly. Phosphorus is a mineral that is vitally important to the normal metabolism of numerous compounds \& (in solution) an acid that, with sulphur, must be neutralized by the base-forming ions of sodium, potassium, calcium, \& magnesium.

About $70 \%$ of retained Phosphorus combines with calcium in bone \& tooth structure. Phosphorus is the principal element in the structure of the nucleus \& cytoplasm of all tissue cells. It is also a universally distributed component of skeletal, nerve, \& muscle tissues.

A reduced concentration of phosphorus in the blood serum is a disorder known as hypophosphatemia. Phosphorus deficiency may cause bone diseases such as rickets in animals.

An improper balance of phosphorus \& calcium may cause osteoporosis. Dietary sources of Phosphorus include milk products, egg yolk, legumes, nuts, \&whole grains.

\section{ETIOLOGY}

1. Deficiency of phosphorous in the diet.

a) Soil deficient in phosphorous.

b) Hay, kadbi and straws are naturally deficient in phosphorous.

c) Drought conditions reduce phosphorous content in the forage.

2. Impaired absorption of phosphorous.

a) Excess calcium, aluminium or iron in diet.

b) Vit. D deficiency.

c) Improper phosphorous and calcium ratio.
3. Increased requirements of phosphorous.

a) Heavy drain of phosphorous through milk in recently calved animals.

b) Increased requirement for development of foetus during advance pregnancy.

4. Feeding cruciferous plants like rape, kale, turnip etc.

TYPE: Acute,Chronicand other.

\section{ACUTE PHOSPHOROUS DEFICIENCY:}

Associated with hypophosphatemia is a wellrecognized problem in high yielding dairy cows at the onset of lactation. The sudden onset of phosphorus losses through the mammary gland at the onset of lactation and decreased feed intake around parturition are believed to be the major contribution to periparturienthypophosphatemia of dairy cows.

\section{CHRONIC PHOSPHOROUS DEFICIENCY:}

Chronic phosphorous deficiencyiscommonly caused by inadequate feed intake or inadequate phosphorus content in the ration over an extended time. This can be seen in grazing animal in arid region with low phosphorus content in soil.

Phosphorus depletion can also result from chronic renal tubular disease due to impaired renal reabsorption of phosphorus or primary hyperparathyroidism causing increased renal phosphorus excretion. Hypophosphatemia is a common finding in horses with chronic renal failure.

\section{HYPOPHOSPHATEMIA \\ PHOSPHOROUS:}

WITHOUT

May occur after oral or parenteral carbohydrate administration \& after parenteral insulin administration as a result of increased cellular phosphorus uptake in combination with glucose. 
Alkalemia\& respiratory alkalosis enhance cellular phosphorus uptake \& therefore also have a hypophosphatemic affect.

\section{CLINICAL FINDINGS:}

ACUTE: phosphorus definition has been associated with anorexia, muscle weakness, muscle \& bone pain, rhabdomyolysis, increased fragility of RBC's \& ensuing intravascular haemolysis.

Other potential effects of hypophosphatemia are neurologic signs presumably related to the altered energy metabolism, impaired cardiac \& respiratory function ( decreased contractility of striated \& Heart muscle ) \& dysfunction of WBCs \& platelets that are believed to be caused by ATP depletion.

In cattle, hypophosphatemia occurring at the onset of lactation is widely believed to be associated with postparturientrecumbency\& the downer cow syndrome.

Downer cow syndrome is occur due to prolong deficiency of phosphorus, calcium, magnesium or potassium in this condition animal is unable to rise even after treatment with calcium salts.

CHRONIC: Signs of chronic phosphorus depletion are most commonly seen in cattle fed a phosphorus deficient diet over several months.

Young animals grow slowly, develop rickets, \& tend to have a rough hair coat, whereas adult animals in early stages may become lethargic, anorectic, \& lose weight.

Decreased milk production \& fertility have erroneously been attributed to phosphorus depletion.

In later stages, animal may develop PICA, osteomalacia (softening of bone), abnormal gait \& lameness, and eventually become recumbent.

PICA in this condition animal eats non-food materials like dirt, paint, hairs and even faecal material.

OTHER: Postparturienthaemoglohinuria is another condition seen in high yielding dairy cows in the first days of lactation. It is characterized by acute intravascular haemolysis \& Haemoglobinuria frequently with fatal outcome and also known as Metabolic or Nutritional haemoglobinuria.

\section{PM FINDINGS :}

In cases of chronic phosphorus depletion are those specific to rickets or osteomalacia.

$>$ Carcasses appear emaciated with a dull hair coat.

$>$ Fractures of ribs, vertebrae, or the pelvis, as well as widened growth plates \& costochondral junction's angular deformities, and shortened long bones are common.

\section{DIAGNOSIS :}

1. HISTORY: History of advance pregnancy / early lactation, sole feeding of dry roughage.

2. SYMPTOMS: Light brown to coffee coloured urine.

3. LESION: Anaemia, jaundice, enlargement of liver and spleen, coffee coloured urine in urinary bladder.

\section{CLINICAL PATHOLOGY:}

a) Haematology: low haemoglobin, PCV and TEC values.

b) Biochemistry: low serum inorganic phosphorous values.

c) Urine analysis: urine positive for haemoglobin.

5. Response to the phosphorous treatment.

6. Determination of the bone density or bone phosphorus content in a rib biopsy has been proposed as a reliable parameter to diagnose chronic phosphorus depletion in cattle.

7. Radiographic example of bone will reveal reduced radiopacity of the bones in chronically

8. Phosphorus depleted animals.

9. Feed samples can be submitted to determine the phosphorus content in ration.

10. In grazing animals, the phosphorus concentration in either soil or in faecal sample can be determined.

\section{TREATMENT:}

\section{SPECIFIC TREATMENT}

I. Animal suffered with phosphorous deficiency can be treated with Sodium acid phosphate (inj. Urimin ) or Buffered phosphorous preparation (inj. Novizac)

(a) $50 \mathrm{ml}$ IV on first day and $25 \mathrm{ml}$ for next 23 days is highly effective. Or 

II. Inj. Tonoricin / tonophosphon @ 30 ml IV or
Inj. Ascorbic acid@15-20 mg/kg. IV daily for 2-4 days also used.

2. SUPPORTIVE TREATMENT: a supportive treatment should also be given to the animal suffered with phosphorous deficiency to maintain normal body functions.

I. Inj. Dextrone $20 \% 500-1000 \mathrm{ml} \mathrm{IV} \mathrm{for} \mathrm{3-4}$ days.

II. Inj. Feritas / Imferon @ 5 ml IM daily for 5-7 days.

III. Inj. Liver extract with B-complex @ 5 ml IM daily for 5-7 days.

IV. Blood transfusion is done in severe cases.

V. Mineral mixture @ 25-50 gm daily orally.

\section{PREVENTION AND CONTROL :}

a) Chronic phosphorous depletion and hypophosphatemia is most effectively treated by providing sufficient amounts of feed with adequate phosphorous content. b) Intravenous administration of phosphorous containing solution is often recommended as the most appropriate approach.

c) In small animals, this is achieved by slow intravenous infusion of sodium phosphate salt solution.

d) Phosphorous depletion in healthy grazing animals is prevented by assuring feed intake with adequate phosphorous content. In animals grazing on phosphorous deficient soils, depletion may be prevented by fertilizing the soils with phosphorous or by supplementing feeds or water supply with phosphorous salts.

e) Feeding higher amount of dietary phosphorous during the last weeks of gestation is contraindicated, because it decreases the intestinal absorption rate of phosphorous and increases therisk of periparturient hypocalcaemia.

f) Regular provision of mineral mixture containing phosphorous according to the requirement for maintenance and production.

g) Protect the recently calved or advanced pregnant animals from stress. 